\title{
Acute kidney injury in trauma patients admitted to the ICU: A systematic review and meta-analysis
}

\section{Running title: Acute kidney injury after trauma in ICU patients}

Signe Søvik, Marie Susanna Isachsen, Kine Marie Nordhuus, Christine Kooy Tveiten, Torsten Eken, Kjetil Sunde, Kjetil Gundro Brurberg, Sigrid Beitland

Søvik: $\quad$ https://orcid.org/0000-0003-4524-2268

Department of Anaesthesia and Intensive Care, Akershus University Hospital, Lørenskog, Norway, \& Institute of Clinical Medicine, Faculty of Medicine, University of Oslo, Oslo, Norway

Isachsen: Medical Library at Ullevål Hospital, University of Oslo Library, Oslo, Norway

Nordhuus: Institute of Clinical Medicine, Faculty of Medicine, University of Oslo, Oslo, Norway

Tveiten: Institute of Clinical Medicine, Faculty of Medicine, University of Oslo, Oslo, Norway

Eken: $\quad$ https://orcid.org/0000-0002-5943-4538

Department of Anaesthesiology, Division of Emergencies and Critical Care, Oslo University Hospital Ullevål, Oslo, Norway \& Institute of Clinical Medicine, Faculty of Medicine, University of Oslo, Oslo, Norway

Sunde: $\quad$ https://orcid.org/0000-0001-9518-5199

Department of Anaesthesiology, Division of Emergencies and Critical Care, Oslo University Hospital Ullevål, Oslo, Norway \& Institute of Clinical Medicine, Faculty of Medicine, University of Oslo, Oslo, Norway

Brurberg: https://orcid.org/0000-0002-5920-4652

Division for Health Services, Norwegian Institute of Public Health, Oslo, Norway Center for Evidence Based Practice, Western Norway University of Applied Sciences, Bergen, Norway

Beitland: https://orcid.org/0000-0001-8803-0105

Department of Anaesthesiology, Division of Emergencies and Critical Care, Oslo University Hospital Ullevål, Oslo, Norway \& Renal Research Group Ullevål, Institute of Clinical Medicine, Faculty of Medicine, University of Oslo, Oslo, Norway

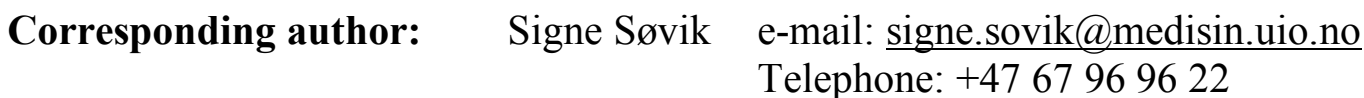

Conflicts of interest: The authors declare that they have no conflicts of interest regarding this study 


\section{"Take-home message"}

Acute kidney injury occurs in $24 \%$ of trauma patients admitted to the intensive care unit, and $10 \%$ of these receive renal replacement therapy. Kidney failure is associated with increased mortality and ICU and hospital length of stay, but renal recovery in survivors is generally good.

\section{0-character Tweet}

Acute kidney injury is common in ICU trauma patients and is associated with increased mortality, but renal recovery in survivors is good. 


\section{Abstract}

Purpose: To perform a systematic review and meta-analysis of acute kidney injury (AKI) in trauma patients admitted to the intensive care unit (ICU).

Methods: We conducted a systematic literature search of studies on AKI according to RIFLE, AKIN or KDIGO criteria in trauma patients admitted to the ICU (PROSPERO CRD42017060420). We searched PubMed, Cochrane Database of Systematic Reviews, UpToDate and NICE through 3 December 2018. Data were collected on incidence of AKI, risk factors, renal replacement therapy (RRT), renal recovery, length of stay (LOS) and mortality. Pooled analyses with random effects models yielded mean differences, OR, and RR, with 95\% CI.

Results: 24 observational studies comprising 25182 patients were included. Study quality (Newcastle-Ottawa scale) was moderate. Study heterogeneity was substantial. Incidence of post-traumatic AKI in the ICU was 24\% (20-29), whereof 13\% (10-16) mild, 5\% (3-7) moderate, and 4\% (3-6) severe AKI. Risk factors for AKI were African American descent, high age, chronic hypertension, diabetes mellitus, high Injury Severity Score, abdominal injury, shock, low Glasgow Coma Scale (GCS), high APACHE II score, and sepsis. AKI patients had 6.0 (4.0-7.9) days longer ICU LOS and increased risk of death (RR 3.4 [2.1-5.7]) compared to non-AKI patients. In patients with AKI, RRT was used in 10\% (6-15). Renal recovery occurred in $96 \%(78-100)$ of patients.

Conclusions: AKI occurred in $24 \%$ of trauma patients admitted to the ICU, with an RRT use among these of $10 \%$. Presence of AKI was associated with increased LOS and mortality, but renal recovery in AKI survivors was good.

\section{Keywords}

Acute kidney injury; wounds and injuries; critical illness; risk factors; mortality; systematic review 


\section{Introduction}

Trauma patients admitted to the intensive care unit (ICU) may develop acute kidney injury (AKI), but the reported incidence of post-traumatic AKI may vary widely depending on the AKI definition used and the study population [1,2]. Consensus definitions of AKI have been developed to include all severities of AKI and to allow comparison between studies. Such definitions include the Risk, Injury, Failure, Loss of kidney function, and End-stage kidney disease (RIFLE) [3], later modified to the Acute Kidney Injury Network (AKIN) [4] and the Kidney Disease: Improving Global Outcomes (KDIGO) criteria [5].

A number of risk factors e.g., hypotension, hypoperfusion, inflammation, critical care medication and rhabdomyolysis have been found to be associated with AKI [1, 2], and several prophylactic strategies have been tested, but it remains unresolved to what degree development of AKI in trauma patients may be prevented [6]. AKI is a heterogeneous condition ranging from mild impairment of kidney function to need of renal replacement therapy (RRT). Clinical data reveal that handling of AKI and use of RRT is strongly variable across ICU departments [7].

AKI in trauma patients is associated with adverse outcomes such as increased length of stay (LOS) and mortality [1, 2]. Survivors of AKI may have variable recovery of kidney function and might be prone to develop chronic kidney disease (CKD) and late morbidity [8] and mortality [9-11]. AKI may also be a burden to the health care system, leading to substantially increased costs especially associated with the use of RRT.

The purpose of the present study was to perform a systematic review and meta-analysis of AKI according to RIFLE, AKIN and/or KDIGO criteria reported in studies of trauma patients admitted to the ICU. Our primary aims were to report on incidence of AKI and compare risk factors and adverse outcomes, such as increased LOS and mortality, in patients with and without post-traumatic AKI. Secondary aims were to report on use of RRT, incidence of renal recovery, and impact on health-care costs. Preliminary results of this study have been presented [12]. 


\section{Methods}

\section{Study registration}

This systematic review and meta-analysis was registered in the PROSPERO database on 12 May 2017 (CRD42017060420). The protocol is available at https://www.crd.york.ac.uk/PROSPEROFILES/60420 PROTOCOL 20170412.pdf. Results were reported according to PRISMA guidelines (Electronic Supplementary Material ESM1). Protocol Appendices 1-3 are ESM2-4.

\section{Literature search}

Papers published between 1 January 2004 and 3 December 2018 were searched by a trained librarian (MSI) in PubMed, Cochrane Database of Systematic Reviews, UpToDate and NICE (National Institute for Health and Care Excellence). Ongoing systematic reviews were identified by searching PROSPERO. In PubMed, Medical Subject Headings (MeSH) and text words including renal insufficiency, acute kidney injury, multiple trauma, nervous system trauma, wounds and injuries, penetrating wounds, accidents, trauma, traumatic, polytrauma, and multiple injuries were searched, alone or in combination. Adapted searches for the other databases, forward citation search using Google Scholar and Web of Science, and hand search of reference lists were conducted.

Inclusion was limited to studies in trauma patients admitted to an ICU, reporting on AKI occurring in the ICU and defined by full or modified RIFLE, AKIN, or KDIGO criteria. The search focused on study population irrespective of intervention, comparison, and outcome. It was limited to English, Swedish, Danish or Norwegian language. See detailed literature search strategy (ESM2).

\section{Study selection}

Two collaborators (KMN and CKT) independently screened studies for eligibility according to pre-defined study selection criteria (ESM3). Titles, abstracts, and keywords from the search were examined, and full text was obtained for all potentially relevant records. Empirical studies comparing AKI and non-AKI patients were included; case reports were excluded. Studies on burns, drownings, and envenomation were excluded. Any disagreement was resolved through discussion with a senior author (SB). 


\section{Data extraction}

Data was extracted in duplicate by two independent collaborators (KMN and CKT) and controlled by two others (SS and SB) according to a pre-defined data extraction form listing all outcomes (ESM4). In cases where data points were missing or ambiguously reported, the first and last author of the study were contacted by e-mail to obtain the data. In case of no reply, one repeated e-mail was sent one month later.

We extracted data on AKI criteria used, incidence of AKI, AKI severity, days to AKI, patient age, gender, African American descent, body mass index (BMI), CKD, diabetes mellitus, chronic hypertension, trauma related risk factors (Injury Severity Score [ISS] [13], Glasgow Coma Scale score (GCS), blunt or penetrating trauma, abdominal injury, presence of shock, rhabdomyolysis), number of packed red blood cell (PRBC) transfusions, use of intravenous starch products and contrast agents, illness severity (Simplified Acute Physiology Score [SAPS II] [14], Acute Physiology And Chronic Health Evaluation [APACHE II and III] score $[15,16]$, mechanical ventilation, multiorgan failure, sepsis), RRT, renal recovery, ICU and hospital LOS, ICU, hospital, and fixed-time mortality. Data was extracted as defined in the included studies, as absolute numbers and means or medians. Data distributions were extracted as standard deviation (SD), standard error (SE), $25^{\text {th }}$ and $75^{\text {th }}$ percentiles, interquartile range (IQR), or $95 \%$ confidence intervals (CI).

\section{Quality assessment}

Two authors (SS and SB) independently and in duplicate assessed the risk of bias of each included study using the Newcastle - Ottawa quality assessment scale [17]. Disagreements were resolved through discussion and by consulting a third author (TE).

\section{Quantitative data synthesis}

\section{Statistical pooling}

Meta-analyses and forest plots were prepared in R [18] using the meta [19] and the forestplot [20] packages. We expected heterogeneity between included studies, hence the meta-analyses were based on a random effect model using the DerSimonian-Laird estimator. Continuous and dichotomous outcomes were compared for patients with and without AKI by calculating mean differences (MD) and risk ratios (RR), respectively. Data primarily reported as medians with IQRs were re-expressed into means and SDs as suggested in the Cochrane handbook [21]. Studies reporting distribution of data only as ranges were not included in the meta-analyses. Meta-analyses of proportions were performed on arcsine-transformed data. 
A number of risk factors potentially associated with the development of AKI were investigated. All risk factors reported in $\geq 3$ primary studies were explored in pooled analyses. A forest plot containing summary estimates for multiple risk factors was generated. Estimates were presented on a common scale, i.e. odds ratio (OR). For dichotomous risk factors, ORs were calculated using the meta package in R. Continuous risk factors were expressed as standardised mean differences (SMDs) using the meta package in R and transformed to OR according to the formula suggested in the Cochrane handbook [22]. Descriptive data are reported as median ( $25 \%-75 \%$ quartiles). For studies reporting mortality at several time points, the measure with longest observation time (up to 90 days) was used in pooled analyses.

\section{Subgroup analyses}

To assess if the results were robust, analyses were conducted in predefined subgroups with different severity levels of AKI, i.e., mild (RIFLE R, AKIN 1, KDIGO 1), moderate (RIFLE I, AKIN 2, KDIGO 2), and severe AKI (RIFLE F, AKIN 3, KDIGO 3). Patients with CKD prior to the trauma (RIFLE L and E) were outside the scope of this meta-analysis. Our protocol included subgroup analysis according to broadly defined trauma mechanisms. After inspecting the included studies we defined the following subgroups: Mixed trauma, iselated traumatic brain injury (TBI), and military casualties. No subgroup analysis according to level of study quality was planned.

\section{Evaluation of heterogeneity}

Statistical heterogeneity among studies was assessed with Cochran's Q test [23] and quantified by the $\mathrm{I}^{2}$ statistic describing the proportion of total variation due to heterogeneity rather than chance $[24,25]$. 


\section{Results}

\section{Study selection}

After removal of duplicates, 1106 studies were identified and their abstracts screened (Fig. 1). Of 278 studies considered potentially eligible, full-text assessment confirmed 34 studies fulfilling the inclusion criteria. In 12 of these publications data were not extractable. Enquiries to authors yielded data from two studies [26, 27], the remaining authors did not respond [2837]. Thus, a total of 24 studies were included in the meta-analysis [1, 2, 26, 27, 38-57].

\section{Study characteristics}

All 24 included studies were observational. One was a pre-planned sub-study in patients recruited to a randomized controlled trial [55], the remaining had cohort design (Table 1). Median (25\%-75\% quartiles) study size was 407 (135-789) patients. Of the total 25182 patients, $77 \%$ were males and 92\% suffered blunt trauma. Age was 42 (34-51) years in AKI patients and 37 (30-42) years in non-AKI patients. Overall prevalence of diabetes mellitus and chronic hypertension was $5 \%$ [38, 42-44, 50, 52, 53] and $16 \%[38,43,44,50,52,53]$, respectively. ISS was 26 (25-28) in AKI patients and 23 (19-25) in non-AKI patients [2, 26, 41, 42, 44-46, 49, 50, 52, 53, 55-57]. APACHE II score was 17 (17-18) in AKI patients and 14 (11-15) in non-AKI patients [1, 27, 40, 44, 46, 49, 51, 55]. Three studies used APACHE III $[40,52,53]$.

Number of studies with our predefined trauma populations for subgroup analyses were 17 with mixed trauma, five with TBI, and two with military trauma (Table 1). RIFLE, AKIN and KDIGO criteria were used in eight studies each. Only 13 of 24 studies used both creatinine and urine output to diagnose AKI; the remaining studies did not use urine output measurements.

\section{Quality assessment}

Overall study quality was moderate (Table 2 ). Eleven studies had generally representative study populations, i.e. unselected mixed trauma patients. All studies had internally representative control groups. Some uncertainty regarding diagnosis of AKI was prevalent: 11 of 24 studies used modified AKI criteria (Table 1) and pre-injury creatinine levels often were unknown, thus exclusion of patients with CKD pre-injury was not ensured. Two studies had short diagnostic windows $[1,50]$ and could have misclassified some patients. Multivariable analysis of risk factors for AKI was performed in 14 of 24 studies. Assessment of outcomes 
(AKI, RRT, LOS, mortality) was overall satisfactory, but only one study explicitly stated that no patients were lost to follow-up [42]. No study was excluded from quantitative analysis due to risk of bias.

\section{Quantitative data synthesis}

\section{Incidence rates}

All 24 studies (25182 patients) reported incidence of AKI among trauma patients in the ICU $[1,2,26,27,38-57]$. Pooled analyses found an overall mean incidence of post-traumatic AKI of 24\% (20-29) (Fig. 2). Time from trauma to AKI diagnosis was three days (range 1-6) [38, 43, 46, 56]. In 22 of 24 studies ( 24630 patients) incidence rates were also reported by AKI severity $[1,2,26,27,38-47,49-51,53-57]$. Among these, 13\% (10-16), 5\% (3-7), and 4\% (36) had mild, moderate, and severe AKI, respectively.

\section{Risk factors}

Risk factors for AKI extracted from the various studies were patient age [1, 2, 26, 27, 38-50, 52-57], male gender [1, 2, 26, 27, 38-50, 52-57], African American descent [26, 40, 46, 52, 53, 56], BMI [40, 52], CKD [44, 52], diabetes mellitus [38, 42-44, 50, 52, 53], chronic hypertension [38, 43, 44, 50, 52, 53], ISS [2, 26, 41, 42, 44-46, 49, 50, 52, 53, 55,-57], SAPS II score [45, 46], APACHE II or III score [1, 27, 40, 44, 46, 49, 51-53, 55], abdominal trauma $[38,45,46,57]$, number of transfused units of PRBC [40, 43, 44, 46, 49, 53], blunt and penetrating trauma $[2,41,42,44,52,53,57]$, shock [38-40, 42, 44, 49, 56, 57], rhabdomyolysis [2, 57], sepsis [38, 42, 57], mechanical ventilation [1, 38, 45, 57], GCS [1, $39,42,43,47,48,50,55]$, acute surgery [43, 52], multiorgan failure [41, 42], and use of intravenous contrast agents $[46,52,53]$ and hydroxyethyl starch [42] products.

Pooled analyses yielded effect estimates for the risk factors associated with increased incidence of post-traumatic AKI (Fig. 3). Abdominal injury and sepsis increased the odds ratio for AKI to more than three. Presence of diabetes mellitus, high APACHE II score, low GCS score, shock, high ISS, and high age gave odds ratios above 2 for AKI. Chronic hypertension and African American descent were also associated with increased risk. Patients given intravenous contrast agents less often developed AKI [46, 52, 53].

\section{Renal replacement therapy}

Use of RRT was reported in 22 of 24 studies (15702 patients) [2, 26, 27, 38-48, 50-57], revealing that it was used in $10 \%(6-15)$ of patients with AKI, corresponding to about $2 \%$ of 
the total trauma population. RRT modes were continuous RRT (CRRT) [42, 44, 46, 55], sustained low efficiency dialysis (SLED) [2], mixed (61\% intermittent haemodialysis (IHD); $39 \%$ CRRT) [57], or unspecified [26, 27, 38, 40, 41, 43, 47, 50-54, 56].

\section{Length of stay}

AKI patients had 6.0 (4.0-7.9) days longer ICU LOS (Fig. 4) [27, 38-42, 44-46, 51, 52, 55] and 5.8 (4.2-7.4) days longer hospital LOS [39-41, 44-46, 55] than non-AKI patients. Association between AKI and increased LOS applied to both the mixed trauma and the TBI subgroups.

\section{Mortality}

Mortality was evaluated at ICU discharge [2, 46, 49, 55], hospital discharge [1, 38-40, 45-48, $53,55,57], 28$ days [44], 30 days [42, 50, 52], 90 days [56], one year [42], or was not specified $[26,41,43,51,54]$. Absolute mortality in AKI patients was 27\% (20-35), but varied considerably (ESM5). Patients with AKI had markedly higher risk of mortality than non-AKI patients (RR 3.4 [2.1-5.7], Fig. 5).

\section{Renal recovery}

Renal recovery occurred in $96 \%(78-100)$ of patients [2, 39, 40, 42, 45, 47, 48, 55, 57] (ESM6). One study reported renal recovery as full or partial [40].

\section{Health-care costs}

None of the included studies reported health-care costs of post-traumatic AKI in the ICU.

\section{Subgroup analyses}

The risk ratio for mortality in AKI patients was 2.9 (1.7-5.1) in mixed trauma, 4.4 (3.1-6.2) in TBI and 8.8 (6.2-12.4) in military trauma patients. Absolute mortality rates in patients with AKI were 29\% (20-38) in mixed trauma, 27\% (8-53) in TBI, and 16\% (9-24) in military trauma.

Mortality data was reported according to AKI severity in 11 of 24 studies (21191 patients) [1, $2,26,27,40,41,45,53,55-57]$. Pooled analyses showed that RR (95\% CI) for death increased with severity of AKI, being 2.8 (1.7-4.6), 5.3 (2.6-10.6), and 6.9 (3.3-14.1) in mild, moderate, and severe AKI, respectively, compared to non-AKI patients. Effect of severity applied to both the mixed and military subgroups. Pooled absolute mortality rates in mild, moderate and severe AKI were 20\% (14-27), 38\% (25-51), and 45\% (31-59) (ESM7, ESM8, ESM9). 


\section{Heterogeneity}

Considerable heterogeneity with $\mathrm{I}^{2}$ above $90 \%$ was observed in some of the meta-analyses.

This can be associated with clinical as well as methodological differences between studies.

Caution is therefore advised when interpreting the results. Specifically, heterogeneity was demonstrated for mortality (Cochran's Q test $\mathrm{p}<0.0001$; Higgins' $\mathrm{I}^{2}=98 \%$ ) and ICU LOS $\left(\mathrm{p}<0.0001 ; \mathrm{I}^{\wedge} 2=85 \%\right)$, but not for hospital $\operatorname{LOS}\left(\mathrm{p}=0.38, \mathrm{I}^{2}=7 \%\right)$. 


\section{Discussion}

This systematic review and meta-analysis reveals that AKI occurs in approximately $24 \%$ of trauma patients admitted to the ICU, a population with a majority of relatively young males suffering blunt trauma. Among these, most patients have less severe AKI and only $4 \%$ have severe AKI. Altogether, less than 2\% of all trauma patients are treated with RRT. Patient related risk factors for post-traumatic AKI are African American descent, high age, chronic hypertension, and diabetes mellitus. Risk factors related to the anatomical injury and physiological response are high ISS, abdominal injury, shock, low GCS, high APACHE II or III score, and sepsis (Fig. 3). Presence of AKI is associated with substantially prolonged ICU and hospital LOS and increased mortality rate that is worsened with the severity of AKI. Kidney function seems to recover well in most trauma patients with AKI, but there is a lack of data on the risk of CKD and long-term mortality. Notably, none of the studies reported on the economic consequences of post-traumatic AKI. Study quality was moderate and heterogeneity was substantial for important outcomes. Our findings should be relevant for healthcare providers, users, and policy makers.

A recent multinational study in a mixed ICU population found that $57 \%$ of patients experienced AKI according to the KDIGO criteria and that $13.5 \%$ were treated with RRT [58]. In contrast, in our systematic review in trauma patients only, about $24 \%$ developed AKI and less than $2 \%$ of the total population was treated with RRT. Post-traumatic AKI is known to represent only a small proportion of severe AKI in the ICU [59], and the subgroup of trauma patients likely differs from other groups of critically ill patients [1]. The trauma population in the present meta-analysis was young and few had chronic hypertension or diabetes mellitus; this may have constituted a larger physiological reserve and reduced the incidence of AKI. However, the incidence of AKI in many of the studies in this systematic review was probably underreported due to the use of modified AKI criteria.

We quantified the effects of many risk factors for post-traumatic AKI, some patient-related and some dependent on the physical injury and its physiological consequences (Fig. 3). From other patient groups it is known that high age, chronic hypertension and diabetes mellitus are risk factors for AKI [58]. In trauma patients a high ISS is a marker of severity [13], while a low GCS score may be associated with hypoventilation and hypoxemia. Shock in trauma patients is usually due to severe bleeding, although other reasons may be present. In the studies reporting abdominal trauma it is unknown whether direct trauma to the kidneys and/or urinary tract affected kidney function. Packing of the abdomen during damage control surgery 
in severe intra-abdominal or retroperitoneal injuries may also affect kidney function. Sepsis in the ICU is known to be a major cause of AKI as it causes both hypoperfusion [60] and inflammatory insult. The APACHE score comprises markers of inflammation, respiratory and circulatory instability, and creatinine levels; a high score was associated with AKI. Patients given intravenous contrast agents developed AKI less often [46, 52, 53], but several restrictions apply to this finding. Neither contrast type, dose, nor concurrent fluid treatment were specified. Also, contrast use may have been avoided in patients thought to be of higher risk of AKI [53].

Unfortunately, we were unable to quantify the impact of several relevant risk factors because they were reported in too few studies (BMI [40, 52], SAPS II [45, 46], multiorgan failure [41, 42], intravenous starch products [42]) or because several studies reported zero events in both groups (mechanical ventilation $[1,38,45,57]$ ).

AKI in ICU patients is associated with high morbidity, and the condition is often part of multiorgan failure [61, 62]. Our observation that patients with AKI had longer ICU LOS and hospital LOS compared to non-AKI patients is therefore expected. The included studies varied widely regarding overall mortality rates and inclusion/exclusion of trauma patients treated in the ICU for less than 24-48 hours. This likely affected the estimated effect on LOS. Although none of the studies in our analysis reported on economic consequences of posttraumatic AKI it is evident that six days extra ICU LOS is associated with increased treatment costs. Use of RRT would add to these expenses due to use of costly equipment and increased work load for the staff [63].

In concordance with previous studies in general ICU populations, we observed that posttraumatic AKI was associated with several-fold increased mortality, worsening with the severity of AKI. Notable exceptions were one study with $>95 \%$ overall mortality [38], one study where non-AKI patients had very high mortality during the initial 48 hours post-injury [45], one study including only traumatic rhabdomyolysis patients [51], and studies with small [49] or elderly patient populations [44]. The increased mortality in trauma patients with AKI is probably multifactorial but is certainly associated with the severity of multiorgan failure. An overall mortality of $27 \%$ in post-traumatic AKI (ESM5) is comparable with what has been observed in a general ICU population [58].

Evaluation of renal recovery across populations is challenging because there is yet no consensus definition [64], thus the definitions may vary from RRT independence via 
normalization of serum creatinine to full recovery of functional reserve. Despite varying definitions in the studies included in the present meta-analysis, renal recovery was reported to occur in $96 \%$ of patients with post-traumatic AKI. However, only one study followed kidney function over a prolonged period of time, and none evaluated the risk of CKD. In other groups of ICU patients it has become evident that an episode of AKI is associated with increased risk of CKD compared to a control group of critically ill patients without AKI [11].

There are important clinical limitations to the studies underlying this systematic review. The external validity of the presented results may be limited because included patient populations in the different studies varied widely with regard to age, comorbidities and trauma mechanisms. None of the studies described their trauma system, in particular formal or informal patient selection processes determining hospital and ICU admission, transfer, and discharge. Thus, possible study bias could not be differentiated from true variation between populations. The estimated incidence of AKI may be confounded by a high overall mortality and high early mortality in some studies. Patients with pre-injury kidney disease were not uniformly excluded in all studies, thus some patients diagnosed with AKI might actually have had CKD. Conversely, most of the studies used modified AKI criteria resulting in systematic underestimation of AKI incidence. Variable and unspecified mortality definitions, especially the evaluation of survival status at administrative instead of fixed time points (e.g., ICU mortality instead of 30-day mortality) is an obvious source of bias since a substantial proportion of trauma deaths occurs after transfer to other ICUs, wards, hospitals, or institutions $[65,66]$. Similarly, the effect of AKI on LOS likely was underestimated since no study reported time spent in other ICUs or hospitals after patient transfer. Use of RRT probably varied across sites as clinical practice depends on local treatment traditions and RRT availability [7]. Data on renal recovery should be interpreted with caution as definitions and assessment time points for this variable varied widely across studies. Unfortunately, we were unable to include data on economic costs because this was not reported in any of the studies.

Methodological limitations include that ten eligible studies could not be included due to unextractable data. As the included studies were observational, associations between risk factors and AKI do not imply a causal relationship. Some publications might have been missed due to the language limitation of our literature search. The strength of evidence varies since variables reported in a high number of primary studies yield better estimates than variables reported in few studies. No study reported rates of missing data; only one study 
reported no loss to follow-up. Possible bias introduced by use of means and SDs for variables that were probably skewed (e.g. durations) was not formally evaluated.

Strengths of this systematic review are the relatively large number of included studies and patients. Further, our literature search, study selection, and data extraction forms were predefined and published before study start. An experienced librarian (MSI) performed the literature search supervised by a consultant intensive care clinician (SB). Screening of studies for eligibility, systematic evaluation of study quality, and data extraction was performed in duplicate by two independent collaborators. For eligible studies without complete and extractable data we contacted authors twice by email in order to retrieve data.

Implications of the present systematic review for future research is a need of studies on posttraumatic AKI, exploring early resuscitative measures, use of RRT, long-term patient outcomes, and treatment costs. There is a clear need for development of uniform standards of reporting in AKI, addressing issues like incidence calculation in populations with high early mortality, definitions of renal recovery, and standardized time points for reporting clinical events such as AKI, renal recovery, and survival status.

In conclusion, the present meta-analysis shows that AKI occurs relatively frequently in trauma patients admitted to the ICU, although severe AKI with need of RRT is uncommon. AKI should be expected and attempted prevented especially in patients with high age, chronic hypertension, diabetes mellitus, severe anatomical injury, and/or marked physiological derangement. Development of post-traumatic AKI is closely associated with increased morbidity and mortality. There is a lack of data on long-term patient outcomes and economic consequences of post-traumatic AKI. 


\section{Funding}

This study was funded by the authors' institutions only. 


\section{Figure Captions}

Fig. 1 Flow chart of search results according to the Preferred reporting items for systematic reviews and meta-analysis (PRISMA). ICU intensive care unit, $A K I$ acute kidney injury

Fig. 2 Proportion of trauma patients admitted to the intensive care unit (ICU) developing acute kidney injury (AKI), overall and split by trauma mechanism. Mild AKI: RIFLE R, AKIN 1, KDIGO 1; Moderate AKI: RIFLE I, AKIN 2, KDIGO 2; Severe AKI: RIFLE F, AKIN 3, KDIGO 3

Fig. 3 Risk factors for acute kidney injury (AKI) in trauma patients admitted to the intensive care unit. The contribution from the various risk factors were statistically weighted and adjusted to a single scale. Odds ratios (OR) for continuous risk factors were derived from standardised mean differences. $C I$ confidence interval, $P R B C$ packed red blood cell, $A P A C H E$ Acute Physiology And Chronic Health Evaluation

Fig. 4 Mean difference in intensive care unit length of stay (LOS, days) in trauma patients with acute kidney injury (AKI) relative to non-AKI trauma patients. $N A K I$ number of AKI patients. $C I$ confidence interval, $R E$ random effects

Fig. 5 Mortality in trauma patients with acute kidney injury (AKI) in the intensive care unit. Risk ratio (RR) of non-survival reported at any time point in patients with AKI, relative to non-AKI trauma patients. $N A K I$ number of AKI patients, $C I$ confidence interval, $R E$ random effects 


\section{Electronic supplementary material (ESM)}

ESM1 PRISMA checklist

ESM2 Literature search strategy

ESM3 Study selection form

ESM4 Data extraction form

ESM5 Absolute mortality (reported at any time point) in trauma patients with acute kidney injury (AKI) in the intensive care unit. $N$ Number of AKI patients, Proportion of nonsurvivors, $C I$ confidence interval, $R E$ random effects

ESM6 Renal recovery (any definition) in trauma patients with acute kidney injury (AKI) in the intensive care unit. $N$ Number of AKI patients, Proportion of patients with renal recovery, $C I$ confidence interval, $R E$ random effects

ESM7 Absolute mortality (reported at any time point) in trauma patients with mild acute kidney injury (Mild AKI) in the intensive care unit. $N$ Number of AKI patients, Proportion of non-survivors, $C I$ confidence interval, $R E$ random effects

ESM8 Absolute mortality (reported at any time point) in trauma patients with moderate acute kidney injury (Moderate AKI) in the intensive care unit. $N$ Number of AKI patients, Proportion of non-survivors, $C I$ confidence interval, $R E$ random effects

ESM9 Absolute mortality (reported at any time point) in trauma patients with severe acute kidney injury (Severe AKI) in the intensive care unit. $N$ Number of AKI patients, Proportion of non-survivors, $C I$ confidence interval, $R E$ random effects 


\section{References}

1. Bagshaw SM, George C, Gibney RTN, Bellomo R (2008) A multi-center evaluation of early acute kidney injury in critically ill trauma patients. Ren Fail 30:581-589. doi: 10.1080/08860220802134649

2. Skinner DL, Hardcastle TC, Rodseth RN, Muckart DJJ (2014) The incidence and outcomes of acute kidney injury amongst patients admitted to a level I trauma unit. Injury 45:259-264. doi: 10.1016/j.injury.2013.07.013

3. Bellomo R, Ronco C, Kellum JA, et al (2004) Acute renal failure - definition, outcome measures, animal models, fluid therapy and information technology needs: the Second International Consensus Conference of the Acute Dialysis Quality Initiative (ADQI) Group. In: Crit Care. pp R204-12

4. Mehta RL, Kellum JA, Shah SV, et al (2007) Acute Kidney Injury Network: report of an initiative to improve outcomes in acute kidney injury. In: Crit Care. p R31

5. Kidney Disease: Improving Global Outcomes KDIGO Acute Kidney Injury Work Group (2012) KDIGO Clinical Practice Guideline for Acute Kidney Injury. Kidney International Supplements 2:1-138. doi: 10.1038/kisup.2012.1

6. Joannidis M, Druml W, Forni LG, et al (2017) Prevention of acute kidney injury and protection of renal function in the intensive care unit: update 2017 : Expert opinion of the Working Group on Prevention, AKI section, European Society of Intensive Care Medicine. Intensive Care Med 43:730-749. doi: 10.1007/s00134-017-4832-y

7. Gatward JJ, Gibbon GJ, Wrathall G, Padkin A (2008) Renal replacement therapy for acute renal failure: a survey of practice in adult intensive care units in the United Kingdom. Anaesthesia 63:959-966. doi: 10.1111/j.1365-2044.2008.05514.x

8. Villeneuve P-M, Clark EG, Sikora L, et al (2016) Health-related quality-of-life among survivors of acute kidney injury in the intensive care unit: a systematic review. Intensive Care Med 42:137-146. doi: 10.1007/s00134-015-4151-0

9. Rimes-StigareIMES-STIGARE C, Awad A, Mårtensson J, et al (2012) Long-term outcome after acute renal replacement therapy: a narrative review. Acta Anaesthesiol Scand 56:138-146. doi: 10.1111/j.1399-6576.2011.02567.x

10. Gallagher M, Cass A, Bellomo R, et al (2014) Long-term survival and dialysis dependency following acute kidney injury in intensive care: extended follow-up of a randomized controlled trial. PLoS Med 11:e1001601. doi: 10.1371/journal.pmed.1001601

11. Chawla LS, Eggers PW, Star RA, Kimmel PL (2014) Acute kidney injury and chronic kidney disease as interconnected syndromes. N Engl J Med 371:58-66. doi: 10.1056/NEJMra1214243

12. Søvik S, Isachsen MS, Nordhuus KM, et al (2018) Acute kidney injury in trauma patients admitted to the intensive care unit: a systematic review and meta-analysis. Conference: 31st Annual Congress of the European Society of Intensive Care Medicine

13. Baker SP, O'Neill B, Haddon W, Long WB (1974) The injury severity score: a method for describing patients with multiple injuries and evaluating emergency care. J Trauma 14:187-196. 
14. Le Gall JR, Lemeshow S, Saulnier F (1993) A new Simplified Acute Physiology Score (SAPS II) based on a European/North American multicenter study. JAMA 270:29572963.

15. Knaus WA, Draper EA, Wagner DP, Zimmerman JE (1985) APACHE II: a severity of disease classification system. Crit Care Med 13:818-829.

16. Knaus WA, Wagner DP, Draper EA, et al (1991) The APACHE III prognostic system. Risk prediction of hospital mortality for critically ill hospitalized adults. Chest 100:16191636. doi: 10.1378/chest.100.6.1619

17. Wells GA, Shea B, OConnell D, et al (2000) The Newcastle-Ottawa Scale (NOS) for assessing the quality of nonrandomised studies in meta-analyses.

http://www.ohri.ca/programs/clinical_epidemiology/oxford.asp. Accessed Jan 01.2019

18. R Core Team (2017) (2017) R: A language and environment for statistical computing. Vienna, Austria

19. Schwarzer G (2015) meta: General Package for Meta-Analysis. R package version 4.3-2.

20. Gordon M, Lumley T (2017) forestplot: Advanced Forest Plot Using "grid" Graphics. R package version 1.7.

21. Higgins J, Deeks JJ (2011) Chapter 7: Selecting studies and collecting data. Cochrane Handbook for Systematic Reviews of Interventions Version 5.1.0

22. Schünemann HJ, Oxman AD, Vist GE, et al (2011) Chapter 12: Interpreting results and drawing conclusions. Cochrane Handbook for Systematic Reviews of Interventions Version 5.1.0

23. Cochran WG (2018) The combination of estimates from different experiments. Biometrics 10:101-129.

24. Higgins JPT, Thompson SG, Deeks JJ, Altman DG (2003) Measuring inconsistency in meta-analyses. BMJ 327:557-560. doi: 10.1136/bmj.327.7414.557

25. Sedgwick P (2015) Meta-analyses: what is heterogeneity? BMJ 350:h1435. doi: 10.1136/bmj.h1435

26. Heegard KD, Stewart IJ, Cap AP, et al (2015) Early acute kidney injury in military casualties. J Trauma Acute Care Surg 78:988-993. doi: 10.1097/TA.0000000000000607

27. Ülger F, Pehlivanlar Küçük M, Küçük AO, et al (2018) Evaluation of acute kidney injury (AKI) with RIFLE, AKIN, CK, and KDIGO in critically ill trauma patients. European Journal of Trauma and Emergency Surgery 44:597-605. doi: 10.1007/s00068-017-08208

28. Baitello AL, Marcatto G, Yagi RK (2013) Risk factors for injury acute renal in patients with severe trauma and its effect on mortality. J Bras Nefrol 35:127-131. doi: $10.5935 / 0101-2800.20130021$

29. Kamar C, Ali A, Altun D, et al (2017) Evaluation of risk factors and development of acute kidney injury in aneurysmal subarachnoid hemorrhage, head injury, and severe sepsis/septic shock patients during ICU treatment. Ulus Travma Acil Cerrahi Derg 23:39-45. doi: 10.5505/tjtes.2016.83451

30. Kim DY, Kobayashi L, Costantini TW, et al (2012) Is contrast exposure safe among the highest risk trauma patients? J Trauma Acute Care Surg 72:61-6- discussion 66-7. doi: 10.1097/TA.0b013e31823f36e0 
31. Leberle R, Ernstberger A, Loibl M, et al (2015) Association of high volumes of hydroxyethyl starch with acute kidney injury in elderly trauma patients. Injury 46:105109. doi: 10.1016/j.injury.2014.08.039

32. Moore EM, Bellomo R, Nichol A, et al (2010) The incidence of acute kidney injury in patients with traumatic brain injury. Ren Fail 32:1060-1065. doi: 10.3109/0886022X.2010.510234

33. Prodhan P, McCage LS, Stroud MH, et al (2012) Acute kidney injury is associated with increased in-hospital mortality in mechanically ventilated children with trauma. J Trauma Acute Care Surg 73:832-837. doi: 10.1097/TA.0b013e31825ab14f

34. Santos PR, Monteiro DLS (2015) Acute kidney injury in an intensive care unit of a general hospital with emergency room specializing in trauma: an observational prospective study. BMC Nephrol 16:30. doi: 10.1186/s12882-015-0026-4

35. Saour M, Charbit J, Millet I, et al (2014) Effect of renal angioembolization on posttraumatic acute kidney injury after high-grade renal trauma: a comparative study of 52 consecutive cases. Injury 45:894-901. doi: 10.1016/j.injury.2013.11.030

36. Talving P, Karamanos E, Skiada D, et al (2013) Relationship of creatine kinase elevation and acute kidney injury in pediatric trauma patients. J Trauma Acute Care Surg 74:912916. doi: 10.1097/TA.0b013e318278954e

37. Zand F, Sabetian G, Abbasi G, et al (2015) Early Acute Kidney Injury based on Serum Creatinine or Cystatin C in Intensive Care Unit after Major Trauma. Iran J Med Sci 40:485-492.

38. de Abreu KLS, Silva Júnior GB, Barreto AGC, et al (2010) Acute kidney injury after trauma: Prevalence, clinical characteristics and RIFLE classification. Indian J Crit Care Med 14:121-128. doi: 10.4103/0972-5229.74170

39. Ahmed M, Sriganesh K, Vinay B, Umamaheswara Rao GS (2015) Acute kidney injury in survivors of surgery for severe traumatic brain injury: Incidence, risk factors, and outcome from a tertiary neuroscience center in India. Br J Neurosurg 29:544-548. doi: 10.3109/02688697.2015.1016892

40. Bihorac A, Delano MJ, Schold JD, et al (2010) Incidence, clinical predictors, genomics, and outcome of acute kidney injury among trauma patients. Ann Surg 252:158-165. doi: 10.1097/SLA.0b013e3181deb6bc

41. Costantini TW, Fraga G, Fortlage D, et al (2009) Redefining renal dysfunction in trauma: implementation of the Acute Kidney Injury Network staging system. J Trauma 67:2837- discussion 287-8. doi: 10.1097/TA.0b013e3181a51a51

42. Eriksson M, Brattström O, Mårtensson J, et al (2015) Acute kidney injury following severe trauma: Risk factors and long-term outcome. J Trauma Acute Care Surg 79:407412. doi: 10.1097/TA.0000000000000727

43. Fang L, You H, Chen B, et al (2010) Mannitol is an independent risk factor of acute kidney injury after cerebral trauma: a case-control study. Ren Fail 32:673-679. doi: $10.3109 / 0886022$ X.2010.486492

44. Fujinaga J, Kuriyama A, Shimada N (2017) Incidence and risk factors of acute kidney injury in the Japanese trauma population: A prospective cohort study. Injury 48:21452149. doi: 10.1016/j.injury.2017.08.022 
45. Gomes E, Antunes R, Dias C, et al (2010) Acute kidney injury in severe trauma assessed by RIFLE criteria: a common feature without implications on mortality? Scand J Trauma Resusc Emerg Med 18:1. doi: 10.1186/1757-7241-18-1

46. Haines RW, Lin S-P, Hewson R, et al (2018) Acute Kidney Injury in Trauma Patients Admitted to Critical Care: Development and Validation of a Diagnostic Prediction Model. Sci Rep 8:3665. doi: 10.1038/s41598-018-21929-2

47. Li N, Zhao W-G, Zhang W-F (2011) Acute kidney injury in patients with severe traumatic brain injury: implementation of the acute kidney injury network stage system. Neurocrit Care 14:377-381. doi: 10.1007/s12028-011-9511-1

48. Li N, Zhao W-G, Xu F-L, et al (2013) Neutrophil gelatinase-associated lipocalin as an early marker of acute kidney injury in patients with traumatic brain injury. J Nephrol 26:1083-1088. doi: 10.5301/jn.5000282

49. Makris K, Markou N, Evodia E, et al (2009) Urinary neutrophil gelatinase-associated lipocalin (NGAL) as an early marker of acute kidney injury in critically ill multiple trauma patients. Clin Chem Lab Med 47:79-82. doi: 10.1515/CCLM.2009.004

50. Podoll AS, Kozar R, Holcomb JB, Finkel KW (2013) Incidence and outcome of early acute kidney injury in critically-ill trauma patients. PLoS ONE 8:e77376. doi: 10.1371/journal.pone.0077376

51. Raju NA, Rao SV, Joel JC, et al (2017) Predictive Value of Serum Myoglobin and Creatine Phosphokinase for Development of Acute Kidney Injury in Traumatic Rhabdomyolysis. Indian J Crit Care Med 21:852-856. doi: 10.4103/ijccm.IJCCM_186_17

52. Reilly JP, Anderson BJ, Mangalmurti NS, et al (2015) The ABO Histo-Blood Group and AKI in Critically Ill Patients with Trauma or Sepsis. Clin J Am Soc Nephrol 10:19111920. doi: 10.2215/CJN.12201214

53. Shashaty MGS, Meyer NJ, Localio AR, et al (2012) African American race, obesity, and blood product transfusion are risk factors for acute kidney injury in critically ill trauma patients. J Crit Care 27:496-504. doi: 10.1016/j.jcrc.2012.02.002

54. Skinner DL, Laing GL, Bruce J, et al (2017) Validating the utilisation of venous bicarbonate as a predictor of acute kidney injury in crush syndrome from sjambok injuries. S Afr Med J 107:446-450. doi: 10.7196/SAMJ.2017.v107i5.12213

55. Skrifvars MB, Moore E, Mårtensson J, et al (2018) Erythropoietin in traumatic brain injury associated acute kidney injury: A randomized controlled trial. Acta Anaesthesiol Scand. doi: 10.1111/aas.13244

56. Stewart IJ, Sosnov JA, Howard JT, Chung KK (2016) Acute Kidney Injury in Critically Injured Combat Veterans: A Retrospective Cohort Study. Am J Kidney Dis 68:564-570. doi: 10.1053/j.ajkd.2016.03.419

57. Yuan F, Hou FF, Wu Q, et al (2009) Natural history and impact on outcomes of acute kidney injury in patients with road traffic injury. Clin Nephrol 71:669-679.

58. Hoste EAJ, Bagshaw SM, Bellomo R, et al (2015) Epidemiology of acute kidney injury in critically ill patients: the multinational AKI-EPI study. Intensive Care Med 41:14111423. doi: 10.1007/s00134-015-3934-7

59. Uchino S, Kellum JA, Bellomo R, et al (2005) Acute renal failure in critically ill patients: a multinational, multicenter study. JAMA 294:813-818. doi: 10.1001/jama.294.7.813 
60. Maheshwari K, Nathanson BH, Munson SH, et al (2018) The relationship between ICU hypotension and in-hospital mortality and morbidity in septic patients. Intensive Care Med. doi: 10.1007/s00134-018-5218-5

61. Lee SA, Cozzi M, Bush EL, Rabb H (2018) Distant Organ Dysfunction in Acute Kidney Injury: A Review. Am J Kidney Dis. doi: 10.1053/j.ajkd.2018.03.028

62. Husain-Syed F, Ricci Z, Brodie D, et al (2018) Extracorporeal organ support (ECOS) in critical illness and acute kidney injury: from native to artificial organ crosstalk. Intensive Care Med 44:1447-1459. doi: 10.1007/s00134-018-5329-z

63. Parikh A, Shaw A (2012) The economics of renal failure and kidney disease in critically ill patients. Crit Care Clin 28:99-111- vii. doi: 10.1016/j.ccc.2011.10.006

64. Forni LG, Darmon M, Ostermann M, et al (2017) Renal recovery after acute kidney injury. Intensive Care Med 43:855-866. doi: 10.1007/s00134-017-4809-x

65. Skaga NO, Eken T, Jones JM, Steen PA (2008) Different definitions of patient outcome: Consequences for performance analysis in trauma. Injury 39:612-622. doi: 10.1016/j.injury.2007.11.426

66. Moore L, Turgeon AF, Emond M, et al (2011) Definition of mortality for trauma center performance evaluation: A comparative study. Crit Care Med 39:2246-2252. doi: 10.1097/CCM.0b013e3182227a59 
Records identified through database searching $(n=1024)$
Additional records identified through other sources $(n=82)$
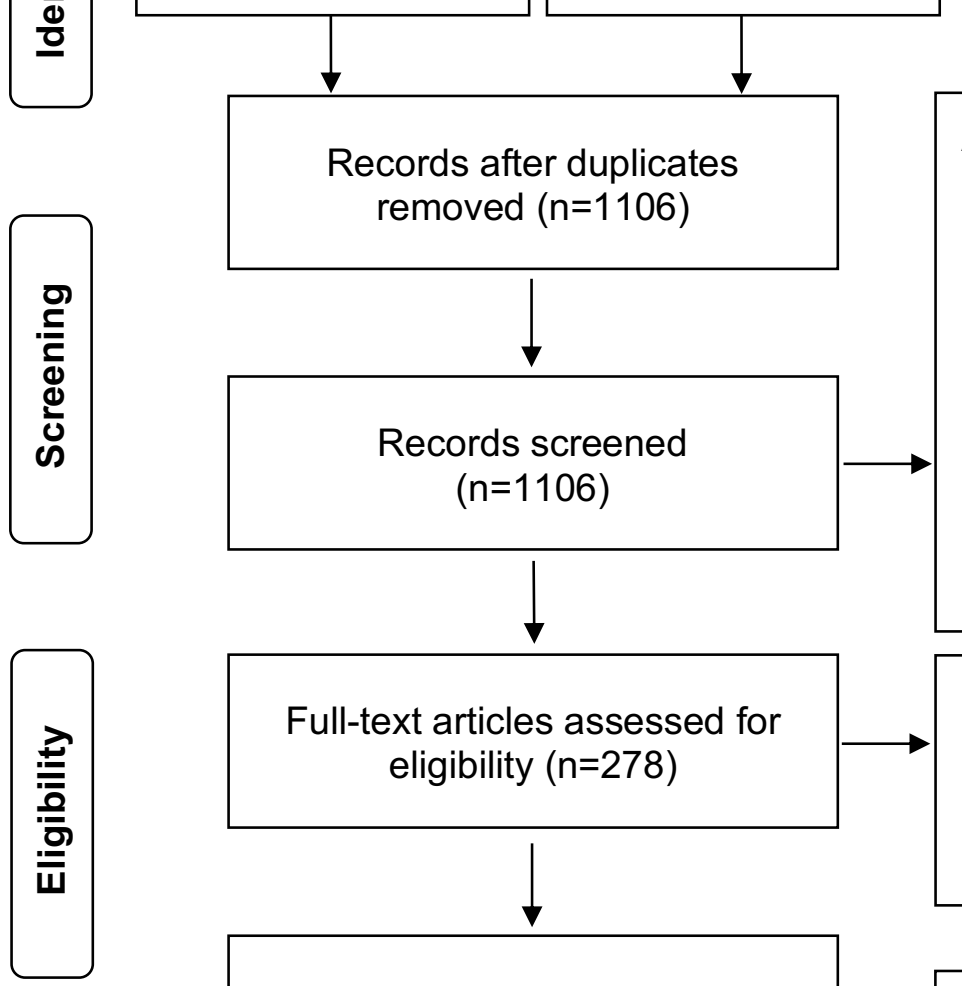

Records after duplicates removed $(n=1106)$

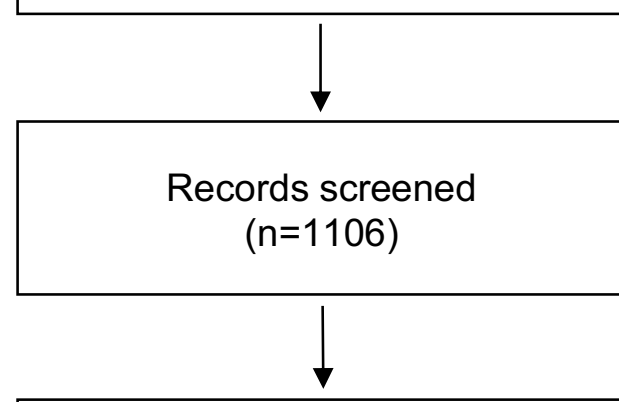

Full-text articles assessed for eligibility $(n=278)$

Full texts excluded $(n=244)$

Not trauma patients $(n=106)$

Not ICU patients $(n=19)$

Not AKI criteria $(n=96)$

Other criteria $(n=23)$

Not humans $(n=26)$

Not trauma patients $(n=67)$

Not ICU patients $(n=49)$

Not AKI $(n=237)$

Not AKI criteria $(n=187)$

Case report $(n=55)$

Letter/comment $(n=67)$

Review/meta-analysis $(n=113)$

Consensus/guideline $(n=8)$

Editorial $(n=16)$

Survey/audit $(n=3)$

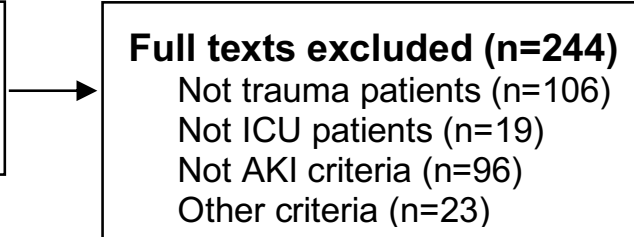

Studies fulfilling inclusion criteria $(n=34)$

Data not extractable $(n=10)$

Studies included in qualitative and quantitative synthesis

$$
(n=24)
$$




\section{Any AKI}

Military trauma, 2 studies, 3941 participants

Mixed trauma, 17 studies, 20181 participants

Brain injury, 5 studies, 1060 participants

Total across subgroups

\section{Mild AKI}

Military trauma, 2 studies, 3941 participants

Mixed trauma, 16 studies, 19984 participants

Brain injury, 4 studies, 1005 participants

Total across subgroups

\section{Moderate AKI}

Military trauma, 2 studies, 3941 participants

Mixed trauma, 16 studies, 19984 participants

Brain injury, 4 studies, 1005 participants

Total across subgroups

\section{Severe AKI}

Military trauma, 2 studies, 3941 participants

Mixed trauma, 16 studies, 19984 participants

Traumatic brain injury, 4 studies, 1005 participants

Total across subgroups
$0.22(0.05$ to 0.46$)$
$0.26(0.20$ to 0.32$)$
$0.20(0.13$ to 0.28$)$
$\mathbf{0 . 2 4}(\mathbf{0 . 2 0}$ to $\mathbf{0 . 2 9})$

$0.17(0.05$ to 0.34$)$

$0.13(0.10$ to 0.17$)$

$0.11(0.08$ to 0.15$)$

0.13 (0.10 to 0.16$)$

0.02 (0.01 to 0.04$)$

0.05 (0.03 to 0.08$)$

0.05 (0.01 to 0.10$)$

0.05 (0.03 to 0.07)

$0.03(0.00$ to 0.09$)$

0.05 (0.03 to 0.07$)$

0.03 (0.01 to 0.05$)$

0.04 (0.03 to 0.06)

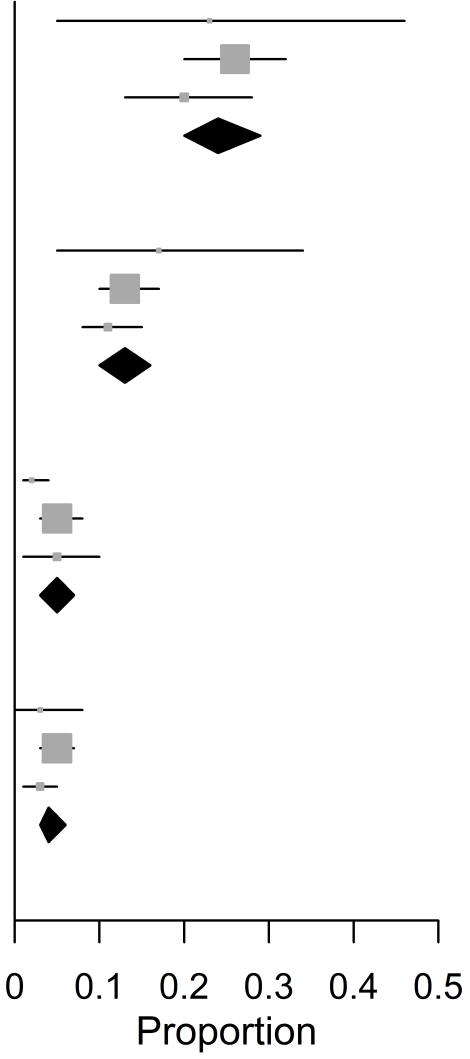




\section{Abdominal injury}

4 studies, 5340 participants, $\left.\right|^{\wedge} 2=81 \%$

Sepsis

3 studies, 4487 participants, $\left.\right|^{\wedge} 2=81 \%$

Diabetes mellitus

7 studies, 2844 participants, $\left.\right|^{\wedge} 2=0 \%$

APACHE II score (high)

7 studies, 11534 participants, $1^{\wedge} 2=67 \%$

Glasgow Coma Scale (low)

8 studies, 11823 participants, $I^{\wedge} 2=92 \%$

Shock

8 studies, 9735 participants, $\left.\right|^{\wedge} 2=72 \%$

PRBC units (high)

4 studies, 2176 participants, $\left.\right|^{\wedge} 2=95 \%$

Injury Severity Score (high)

13 studies, 13167 participants, $\left.\right|^{\wedge} 2=97 \%$

\section{Age (high)}

22 studies, 24692 participants, $I^{\wedge} 2=89 \%$

Chronic hypertension

6 studies, 2431 participants, $\left.\right|^{\wedge} 2=5 \%$

African American descent

6 studies, 6650 participants, $I^{\wedge} 2=0 \%$

Male gender

23 studies, 24687 participants, I^$^{\wedge}=79 \%$

\section{Blunt trauma}

7 studies, 6825 participants, $I^{\wedge} 2=60 \%$

I.v. contrast agents

3 studies, 1727 participants, $\left.\right|^{\wedge} 2=83 \%$
3.1 (1.7 to 5.5$)$

3.0 (1.6 to 5.6$)$

2.4 (1.7 to 3.4$)$

2.4 (1.8 to 3.2$)$

2.3 (1.3 to 4.0$)$

2.3 (1.6 to 3.2$)$

2.1 (0.8 to 5.5$)$

2.1 (1.2 to 3.6$)$

2.1 (1.7 to 2.6)

1.8 (1.3 to 2.3$)$

$1.6(1.3$ to 2.0$)$

$1.3(1.0$ to 1.6$)$

0.8 (0.6 to 1.3$)$

$0.5(0.3$ to 1.0$)$

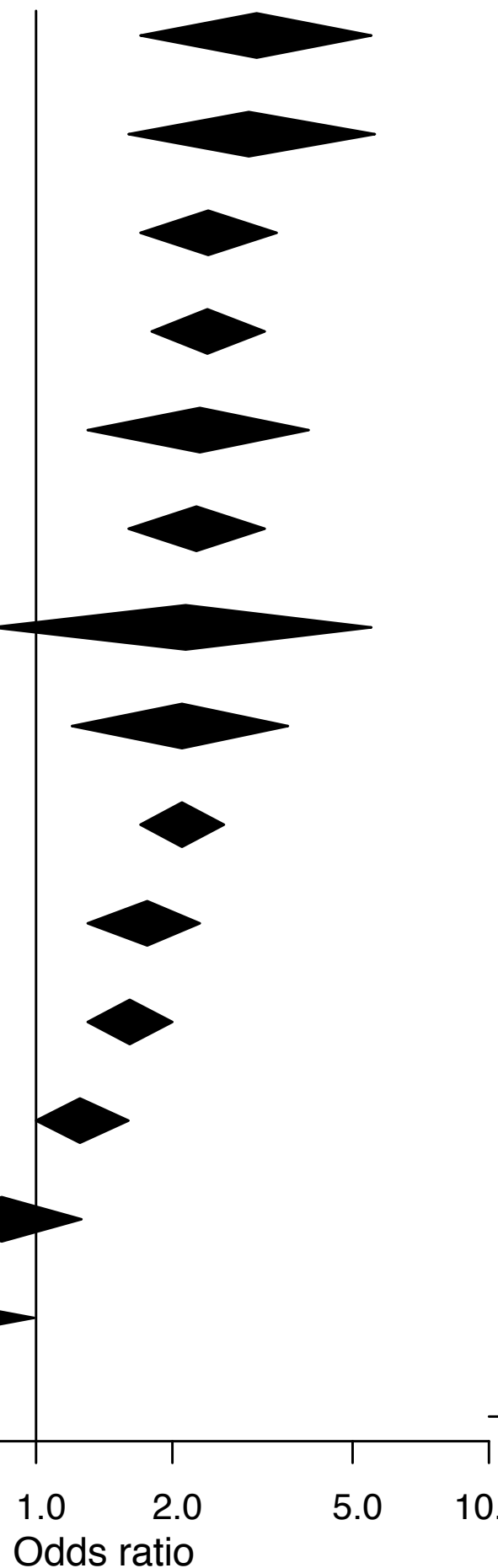




\section{Study}

Constatini '09

Bihorac '10 de Abreu '10 Gomes '10 Ahmed '15 Eriksson'15 Reilly '15 Fujinaga '17 Raju '17 Ülger'17 Haines '18 Skifvars '18

Overall
N AKI LOS $\quad 95 \%$ Cl Weight (\%) Mean difference (RE model)

$\begin{array}{rrcr}170 & 6.6 & {[4.5 ; 8.7]} & 11.0 \\ 253 & 9.0 & {[7.1 ; 10.9]} & 11.2 \\ 52 & -5.0 & {[-38 ; 28]} & 0.3 \\ 217 & 4.0 & {[2.6 ; 5.4]} & 11.9 \\ 11 & 10.0 & {[1.0 ; 19.0]} & 3.4 \\ 103 & 7.0 & {[5.1 ; 8.9]} & 11.3 \\ 134 & 13.0 & {[4.2 ; 21.8]} & 3.5 \\ 66 & 3.0 & {[1.5 ; 4.5]} & 11.8 \\ 14 & 6.7 & {[-3.1 ; 16.5]} & 2.9 \\ 147 & 10.0 & {[7.8 ; 12.2]} & 10.9 \\ 163 & 2.0 & {[0.5 ; 3.5]} & 11.8 \\ 82 & 3.0 & {[0.2 ; 5.8]} & 9.9\end{array}$

$1412 \quad 6.0 \quad[4.0 ; 7.9] \quad 100 \%$
Heterogeneity: |^2=85\%, tau^2=7.545, $p<0.0001$ Test for overall effect: $p<0.0001$

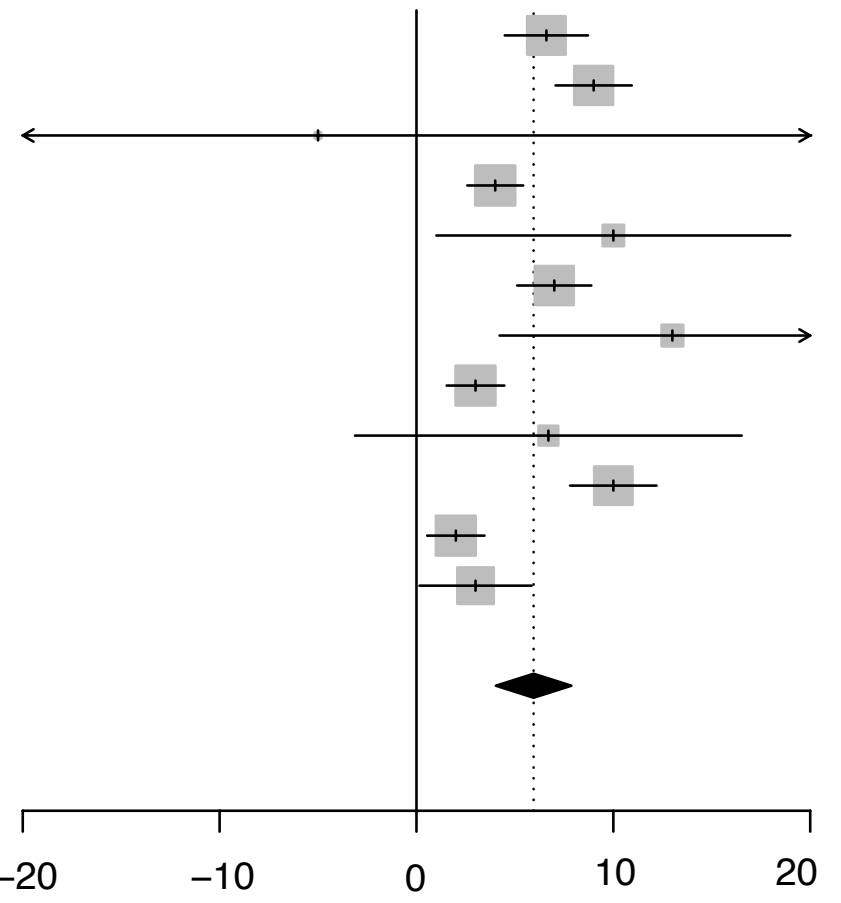

Days in ICU 


\section{Study}

Bagshaw '08

Yuan '09

Constatini '09

Makris '09

Bihorac '10

de Abreu '10

Gomes '10

Li '11

Shashaty '12

Li '13

Podoll '13

Ahmed '15

Eriksson '15

Heegard '15

Reilly '15

Stewart '16

Fujinaga '17

Raju '17

Skinner'17

Ülger '17

Haines '18

Skifvars '18

\section{N AKI RR $\quad 95 \%$ Cl Weight (\%) Risk ratio (RE model)}

\begin{tabular}{|c|c|c|c|c|}
\hline 1711 & 2.1 & [1.9; & 2.4] & 5.4 \\
\hline 423 & 7.5 & [6.5; & 8.7] & 5.4 \\
\hline 170 & 2.9 & [1.7; & 4.9] & 5.1 \\
\hline 11 & 2.4 & [0.7; & 8.9] & 4.0 \\
\hline 253 & 5.9 & [4.1; & 8.4] & 5.3 \\
\hline 52 & 1.0 & [1.0; & 1.1] & 5.4 \\
\hline 217 & 0.6 & {$[0.4 ;$} & $0.8]$ & 5.3 \\
\hline 31 & 5.2 & {$[2.7 ;$} & $10.0]$ & 5.0 \\
\hline 147 & 3.8 & [1.8; & 7.8] & 4.9 \\
\hline 13 & 5.4 & {$[1.5 ;$} & $19.5]$ & 4.0 \\
\hline 54 & 3.7 & [2.3; & 6.0] & 5.2 \\
\hline 11 & & & & 0.0 \\
\hline 103 & 3.0 & [1.6; & 5.6] & 5.0 \\
\hline 46 & 9.6 & [2.2; & 41.8] & 3.7 \\
\hline 134 & 4.9 & [2.7; & 8.9] & 5.1 \\
\hline 474 & 8.7 & [6.1; & 12.5] & 5.3 \\
\hline 66 & 2.0 & {$[0.4 ;$} & $10.8]$ & 3.4 \\
\hline 14 & 1.2 & {$[0.5 ;$} & 2.6] & 4.8 \\
\hline 46 & 23.0 & [2.6; & 201] & 2.7 \\
\hline 147 & 6.8 & {$[2.2 ;$} & 20.8] & 4.3 \\
\hline 163 & 2.1 & [1.6; & 2.8] & \\
\hline 82 & 4.0 & [2.6; & $6.1]$ & 5.3 \\
\hline
\end{tabular}

$4368 \quad 3.4 \quad[2.1 ; 5.7] \quad 100 \%$

Overall

Heterogeneity: I^2=98\%, tau^2=1.182, $p<0.0001$

Test for overall effect: $p<0.0001$

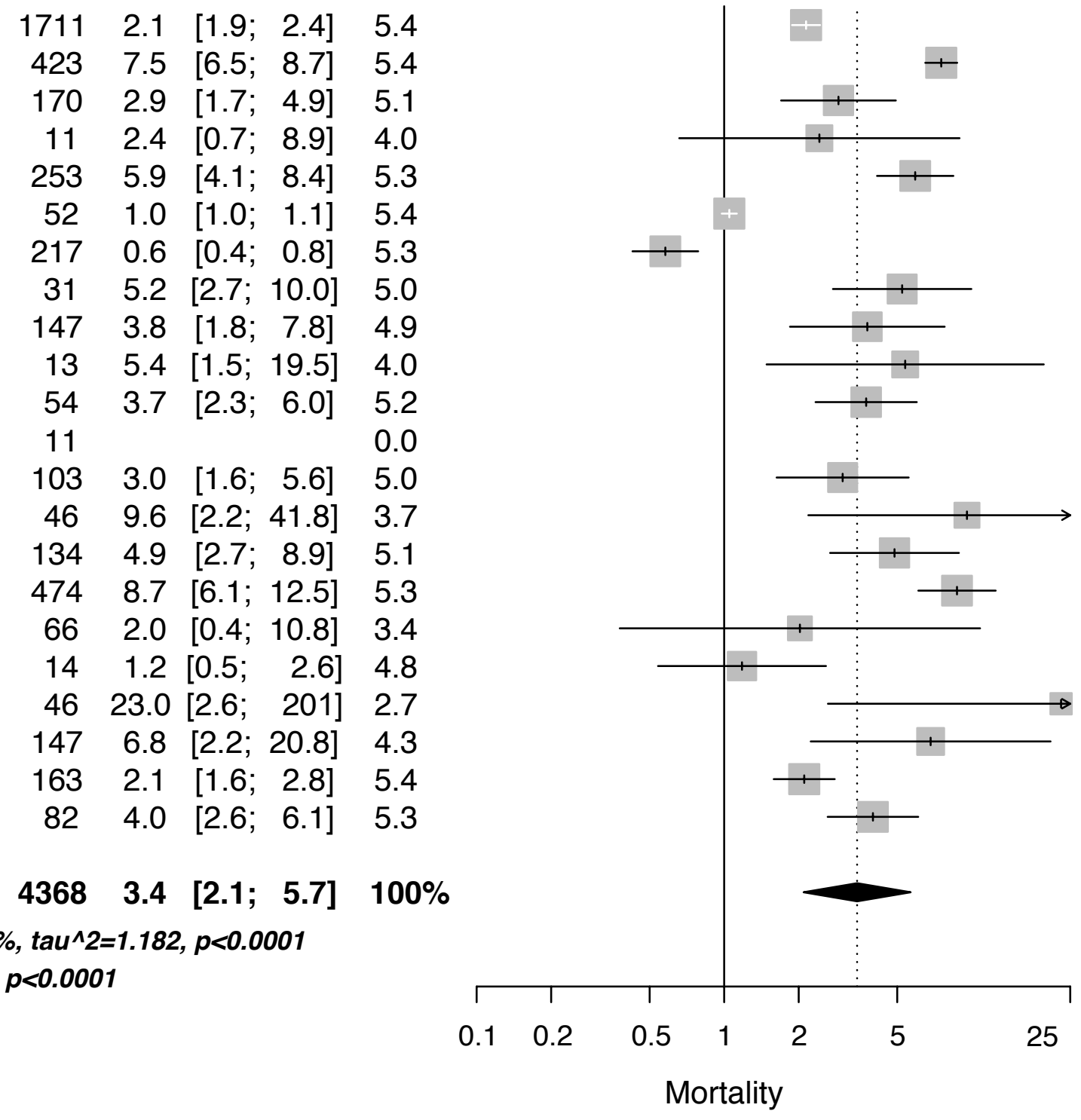


Table 1: Included studies of acute kidney injury (AKI) in ICU trauma patients

\begin{tabular}{|c|c|c|c|c|c|c|c|c|}
\hline \multicolumn{2}{|c|}{$\begin{array}{l}\text { First author } \\
\text { Publication year }\end{array}$} & \multirow{2}{*}{$\begin{array}{l}\text { AKI } \\
\text { Criteria }\end{array}$} & \multirow{2}{*}{$\begin{array}{l}\begin{array}{l}\text { Criteria } \\
\text { adherence }\end{array} \\
\text { Modified }^{a}\end{array}$} & \multirow{2}{*}{$\begin{array}{l}\text { Population } \\
\text { Mixed Trauma }\end{array}$} & \multirow{2}{*}{$\begin{array}{l}\text { Recruitment } \\
\text { RCS }\end{array}$} & \multirow{2}{*}{$\begin{array}{l}\text { AKI } \\
\text { follow-up time } \\
24 \text { hours }\end{array}$} & \multirow{2}{*}{$\begin{array}{r}\mathbf{N} \\
\text { Total } \\
9449\end{array}$} & \multirow{2}{*}{$\begin{array}{r}\text { N (\%) } \\
\text { with AKI } \\
1711 \text { (18) }\end{array}$} \\
\hline Bagshaw ‘08 & [1] & & & & & & & \\
\hline Yuan '09 & [57] & RIFLE & Modified $^{a}$ & Mixed Trauma & RCS & In-hospital & 3945 & $423(11)$ \\
\hline Constatini '09 & [41] & AKIN & Original & Mixed Trauma & RCS & Unspecified & 571 & $170(30)$ \\
\hline Makris '09 & [49] & RIFLE & Original & Mixed Trauma & PCS & 5 days & 31 & $11(36)$ \\
\hline Bihorac '10 & [40] & RIFLE & Modified & Mixed Trauma & PCS & 28 days & 982 & $253(26)$ \\
\hline de Abreu '10 & [38] & RIFLE & Modified & Mixed Trauma & RCS & Hospital stay & 129 & $52(40)$ \\
\hline Fang '10 & [43] & RIFLE & Modified & TBI & RCS & Not specified & 171 & $53(31)$ \\
\hline Gomes '10 & [45] & RIFLE & Original ${ }^{a}$ & Mixed Trauma & PCS & ICU stay & 436 & $217(50)$ \\
\hline Li'11 & [47] & AKIN & Original & TBI & RCS & In-hospital & 136 & $31(23)$ \\
\hline Shashaty '12 & [53] & AKIN & Modified & Mixed Trauma & PCS & 5 days & 400 & $147(37)$ \\
\hline Li'13 & [48] & AKIN & Original & TBI & PCS & Hospital stay & 55 & $13(24)$ \\
\hline Podoll '13 & [50] & AKIN & Modified & Mixed Trauma & RCS & 72 hours & 901 & $54(6)$ \\
\hline Skinner '14 & [2] & RIFLE & Modified & Mixed Trauma & RCS & Hospital stay & 666 & $102(15)$ \\
\hline Ahmed '15 & [39] & AKIN & Original & TBI & RCS & Hospital stay & 95 & $11(12)$ \\
\hline Eriksson '15 & [42] & KDIGO & Modified ${ }^{a}$ & Mixed Trauma & PCS & 1 year & 413 & $103(25)$ \\
\hline Heegard '15 & [26] & KDIGO & Modified a & Military & PCS & 14 days & 134 & $46(34)$ \\
\hline Reilly'15 & [52] & AKIN & Modified & Mixed Trauma & PCS & 6 days & 497 & $134(27)$ \\
\hline Stewart '16 & [56] & KDIGO & Modified a & Military & RCS & 7 days & 3807 & $474(13)$ \\
\hline Fujinaga '17 & [44] & KDIGO & Original a & Mixed Trauma & PCS & ICU stay & 333 & $66(20)$ \\
\hline Raju '17 & [51] & AKIN & Unknown & Mixed Trauma & PCS & Not specified & 90 & $14(16)$ \\
\hline Skinner '17 & [54] & KDIGO & Modified $^{a}$ & Mixed Trauma & RCS & Not specified & 310 & $46(15)$ \\
\hline Ülger '17 & [27] & KDIGO & Original a & Mixed Trauma & RCS & Not specified & 198 & $147(74)$ \\
\hline Haines '18 & [46] & KDIGO & Modified & Mixed trauma & RCS & 7 days & 830 & $163(20)$ \\
\hline Skrifvars '18 & [55] & KDIGO & Original & TBI & RCT & 7 days & 603 & $82(14)$ \\
\hline
\end{tabular}


RIFLE Risk, injury, failure, loss, end-stage, AKIN Acute Kidney Injury Network, KDIGO Kidney disease: Improving global outcomes. Modified Urine output not included, aBaseline creatinine levels estimated by formulae if missing in patient records. TBI Traumatic brain injury, RCT Randomised controlled trial, PCS

Prospective cohort study, RCS Retrospective cohort study 
Table 2: Quality assessment of studies according to the Newcastle - Ottawa quality assessment scale

\begin{tabular}{|c|c|c|c|c|c|c|c|c|c|}
\hline \multicolumn{2}{|c|}{$\begin{array}{l}\text { First Author } \\
\text { Publication year }\end{array}$} & \multirow{2}{*}{$\begin{array}{l}\text { Represent- } \\
\text { ativeness } \\
\text { (a) } \\
\text { A }\end{array}$} & \multirow{2}{*}{$\begin{array}{l}\text { Selection of } \\
\text { non-exposed } \\
\text { (b) }\end{array}$} & \multirow{2}{*}{$\begin{array}{l}\text { Ascertain- } \\
\text { ment of } \\
\text { exposure (c) } \\
\text { A }\end{array}$} & \multirow{2}{*}{$\begin{array}{l}\begin{array}{l}\text { Incident } \\
\text { disease (d) }\end{array} \\
\text { B }\end{array}$} & \multirow{2}{*}{$\begin{array}{l}\begin{array}{l}\text { Compara- } \\
\text { bility (e) }\end{array} \\
\text { A }\end{array}$} & \multirow{2}{*}{$\begin{array}{l}\begin{array}{l}\text { Assessment } \\
\text { of outcome (f) }\end{array} \\
\text { A }\end{array}$} & \multirow{2}{*}{$\begin{array}{l}\begin{array}{l}\text { Length of } \\
\text { follow-up (g) }\end{array} \\
\text { B }\end{array}$} & \multirow{2}{*}{$\begin{array}{l}\begin{array}{l}\text { Adequacy of } \\
\text { follow-up (h) }\end{array} \\
D\end{array}$} \\
\hline Bagshaw ‘08 & [1] & & & & & & & & \\
\hline Yuan '09 & [57] & A & A & A & A & A & A & A & $\mathrm{D}$ \\
\hline Constatini ‘09 & [41] & $A$ & $A$ & $A$ & $A$ & $\mathrm{C}$ & $A$ & $A$ & $D$ \\
\hline Makris ‘09 & [49] & $B$ & $A$ & $A$ & $A$ & C & $A$ & $A$ & $\mathrm{D}$ \\
\hline Bihorac ‘10 & [40] & B & $A$ & $A$ & $A$ & $A$ & $A$ & A & $\mathrm{D}$ \\
\hline de Abreu '10 & [38] & C & $A$ & $A$ & $B$ & $\mathrm{C}$ & $A$ & A & $\mathrm{D}$ \\
\hline Fang '10 & [43] & $\mathrm{C}$ & $A$ & $A$ & $B^{i}$ & $A$ & $A$ & A & $\mathrm{D}$ \\
\hline Gomes '10 & [45] & $A$ & $A$ & $A$ & $A$ & C & $A$ & A & $\mathrm{D}$ \\
\hline Li'11 & [47] & $\mathrm{C}$ & $A$ & $A$ & $A$ & $\mathrm{C}$ & $A$ & $A$ & $\mathrm{D}$ \\
\hline Shashaty '12 & [53] & $A$ & $A$ & $A$ & $B^{i}$ & $A$ & $A$ & $A$ & $\mathrm{D}$ \\
\hline $\mathrm{Li} 13$ & [48] & C & $A$ & $A$ & $A$ & $\mathrm{C}$ & $A$ & $A$ & $\mathrm{D}$ \\
\hline Podoll ‘13 & [50] & $A$ & $A$ & $A$ & $A$ & $A$ & $A$ & $A$ & $\mathrm{D}$ \\
\hline Skinner '14 & [2] & C & $A$ & $A$ & B & $A$ & $A$ & $A$ & $\mathrm{D}$ \\
\hline Ahmed '15 & [39] & C & A & A & A & C & A & A & D \\
\hline Eriksson '15 & [42] & A & $A$ & $A$ & $A$ & $A$ & $A$ & $A$ & $A$ \\
\hline Heegard '15 & [26] & C & A & A & $\mathrm{B}^{\mathrm{k}}$ & A & $A$ & A & D \\
\hline Reilly ‘15 & [52] & $A$ & $A$ & $A$ & $B^{i}$ & $A$ & $A$ & $A$ & $D$ \\
\hline Stewart ‘16 & [56] & $\mathrm{C}$ & $A$ & $A$ & $\mathrm{~B}^{\mathrm{k}}$ & A & $A$ & $A$ & $\mathrm{D}$ \\
\hline Fujinaga '17 & [44] & $A$ & $A$ & $A$ & $B^{i}$ & $A$ & $A$ & $A$ & $D$ \\
\hline Raju'17 & [51] & $B$ & $A$ & $A$ & $A$ & C & $A$ & $A$ & $\mathrm{D}$ \\
\hline Skinner '17 & [54] & $C$ & $A$ & $A$ & B & C & $A$ & $B$ & $D$ \\
\hline Ülger ‘17 & [27] & $A$ & $A$ & $A$ & $A$ & $\mathrm{C}$ & $A$ & $A$ & $\mathrm{D}$ \\
\hline Haines '18 & [46] & $A$ & $A$ & $A$ & $A$ & $A$ & $A$ & $A$ & $D$ \\
\hline Skrifvars '18 & [55] & $\mathrm{C}$ & $A$ & $A$ & $A$ & $A$ & $A$ & $A$ & $D$ \\
\hline
\end{tabular}

a) A, truly representative; $B$, somewhat representative; $C$, selected group; D, no description of the derivation of the cohort. b) $A$, drawn from the same community as the exposed; B, drawn from a different source; $C$, no description of the derivation of the non-exposed. c) A, secure record; B, structured interview; C, written self-report; D, no description. d) Demonstration that the outcome of interest was not present at start of study: A, yes; B, no.

e) A, study controls for demographics / co-morbidities; B, study controls for any additional factor (e.g., age, severity of illness); C, not done. f) A, independent or blind assessment; B, record linkage; C, self-report; D, no description. g) Long enough for outcomes to occur? A, yes, (i.e. in-hospital or up to $30 \mathrm{~d}$ ); B, no.

h) A, complete follow-up; B, subjects lost to follow-up was unlikely to introduce bias; C, follow-up rate $90 \%$ or lower; D, no statement. Only one study explicitly 
stated complete follow-up. All studies reported complete numbers on mortality. ${ }^{i}$ Only patients on chronic hemodialysis were excluded. ${ }^{k}$ Not described; combat casualties who were unlikely to have chronic kidney disease 
Click here to access/download Supplementary Material ESM-1 PRISMA checklist.pdf 
Click here to access/download Supplementary Material ESM-2 LIterature search strategy.pdf 
Click here to access/download Supplementary Material ESM-3 Study selection form.pdf 
Click here to access/download Supplementary Material ESM-4 Data extraction form.pdf 
Click here to access/download
Supplementary Material

Click here to access/download
Supplementary Material ESM-5-6-7-8-9.pdf 